\title{
A pilot and feasibility study of the effectiveness of care mapping on person-centred care in neurorehabilitation settings
}

DOI:

10.1080/09602011.2019.1598446

\section{Document Version}

Accepted author manuscript

Link to publication record in Manchester Research Explorer

Citation for published version (APA):

Brown, L., Blake, D., Berry, K., \& Sheldrick, R. (2019). A pilot and feasibility study of the effectiveness of care mapping on person-centred care in neurorehabilitation settings. Neuropsychological Rehabilitation. https://doi.org/10.1080/09602011.2019.1598446

\section{Published in:}

Neuropsychological Rehabilitation

\section{Citing this paper}

Please note that where the full-text provided on Manchester Research Explorer is the Author Accepted Manuscript or Proof version this may differ from the final Published version. If citing, it is advised that you check and use the publisher's definitive version.

\section{General rights}

Copyright and moral rights for the publications made accessible in the Research Explorer are retained by the authors and/or other copyright owners and it is a condition of accessing publications that users recognise and abide by the legal requirements associated with these rights.

\section{Takedown policy}

If you believe that this document breaches copyright please refer to the University of Manchester's Takedown Procedures [http://man.ac.uk/04Y6Bo] or contact uml.scholarlycommunications@manchester.ac.uk providing relevant details, so we can investigate your claim.

\section{OPEN ACCESS}




\section{A pilot and feasibility study of the effectiveness of care mapping on person- centred care in neurorehabilitation settings}

This is a pre-copyedited, author-produced PDF of an article accepted for publication

in Neuropsychological Rehabilitation following peer review. The final published version of the article Brown et al (2019) will be available from the Journal website:

https://www.tandfonline.com/toc/pnrh20/current

Laura J.E. Brown ${ }^{\mathrm{a} *}$, Daniel Blake ${ }^{\mathrm{a}}$, Katherine Berry ${ }^{\mathrm{a}}$, and Russell Sheldrick ${ }^{\mathrm{b}}$

${ }^{a}$ Division of Psychology and Mental Health, School of Health Sciences, Faculty of Biology, Medicine and Health, University of Manchester, Manchester Academic Health Science Centre, Manchester, United Kingdom.

${ }^{b}$ Department of Clinical Neuropsychology, Salford Royal NHS Foundation Trust, Salford, United Kingdom

Please address all correspondence to:

Dr Laura Brown

Division of Psychology and Mental Health, School of Health Sciences

The University of Manchester

Room 2.32, Second Floor, Zochonis Building

Brunswick Street

Manchester M13 9PL

+44(0)1612752563

laura.brown@manchester.ac.uk 


\section{A pilot and feasibility study of the effectiveness of care mapping on person- centred care in neurorehabilitation settings}

Dementia Care Mapping for Neurorehabilitation (DCM-NR) is a tool designed to increase person centred care (PCC) in neurorehabilitation settings. This paper reports pilot and feasibility testing of a cluster-randomised controlled trial assessing the effectiveness of DCM-NR at increasing PCC. Forty-one staff members, from four neurorehabilitation wards, were cluster-randomised to receive DCM-NR (experimental group) or care as usual (control). Measures of PCC, attitudes to people with brain injury, and PCC self-efficacy were taken at baseline and follow-up. The experimental group were also interviewed about their experiences of the trial. Twenty-nine participants completed the study. Participants generally found the intervention and trial to be acceptable. PCC was significantly higher in the experimental than the control group at follow-up, despite levels of exposure to the intervention being low. PCC-self efficacy increased in the control group, but not in the experimental group, perhaps reflecting an initial reduction in perceived competence following feedback. Neither group showed a change in attitudes to people with brain injury. The findings suggest that this cluster-randomised trial design is acceptable; that DCM-NR shows promise as a tool for increasing PCC; and highlight modifications to the protocol that could increase the success and theoretical value of a future large-scale study.

Keywords: Dementia Care Mapping; DCM; DCM-NR; person-centred care; feasibility; RCT; pilot; neurorehabilitation 


\section{Introduction}

Delivering Person-Centred Care (PCC) is a core requirement for supporting people with longterm neurological conditions (Department of Health, 2005). Dementia Care Mapping (DCM) is an observational tool designed to increase levels of PCC. As the name implies, it was originally developed for improving care for people with dementia (Brooker, 2005). However, it has since been adapted for use with other populations, including those in neurorehabilitation settings (McIntosh et al., 2012). Kuhn, Ortigata and Kasayaka (2000) describe how a typical DCM session involves one or more trained mappers continuously observing a small number of care recipients in communal parts of the facilities over a number of hours. The experiences of each care recipient are coded according to a list of pre-specified behaviours (such as 'eating or drinking' or 'engaging in self-care'), and scored according to the extent that each activity or interaction is likely to improve or detract from the individual's wellbeing. The results of the mapping are then fed back at a group-level to staff so that ways of improving care practices can be discussed, and an action plan developed. The adapted DCM for Neurorehabilitation settings (DCM-NR) follows a similar protocol, but includes slight modifications to the coding framework, such as the inclusion of additional neurorehabilitation-specific behavioural codes for recording medical interventions or activities relating to therapeutic treatment (O’Hanlon et al., 2014).

Previous work has shown DCM-NR to be a valid and acceptable tool for use in neurorehabilitation settings. For instance, Westbrook et al (2013) investigated the acceptability of DCM-NR to staff and patients in a 12-bed acute neurorehabilitation ward. Members of staff generally reported that the process felt worthwhile and not burdensome, and the majority of patients agreed that it was a good way to assess care, and did not feel that the observations were intrusive or made them uncomfortable. O'Hanlon et al (2014) also showed that DCM-NR has good validity and reliability as a measure of PCC in neurorehabilitation settings.

DCM has previously been shown to increase levels of PCC in dementia settings (Martin \& Younger, 2001). However, the efficacy of DCM-NR at increasing levels of PCC in neurorehabilitative settings has not yet been investigated. Before implementing a full scale evaluation of a complex intervention, appropriate feasibility and pilot testing of the evaluative procedures needs to be carried out (Medical Research Council, 2006). These allow important procedural metrics, such as participant recruitment and retention rates, and the variability of outcome measures, to be estimated in advance and used for planning the fullscale trial. Factors that might affect the fidelity of implementation, such as levels of exposure and responsiveness to the intervention (Carroll et al., 2007) or contamination of the intervention to the control group (Dane \& Schneider (1998), and any unintended consequences of the trial, can also be identified, and later addressed. Hypotheses about the potential mechanisms underlying any changes in outcome measures can also be made, and then subsequently tested in a full-scale trial.

The aims of the current study were therefore to conduct pilot and feasibility testing of a cluster-randomised controlled trial to assess the effectiveness of DCM-NR at increasing 
levels of PCC. Members of staff from four neurorehabilitation wards of the same hospital were cluster-randomised to receive either DCM-NR or to continue with care as usual. Levels of PCC were quantified using the Quality of Interactions Schedule (QUIS: Dean, Proudfoot $\&$ Lindesay, 1993). Given the known roles of staff attitudes and beliefs in shaping the clinical practice of healthcare professionals (Godin, Bélanger-Gravel \& Grimshaw, 2008), staff members' attitudes towards people with neurological conditions, and their self-efficacy in delivering PCC, were also assessed. The specific objectives of the study were to: (i) estimate recruitment and retention rates of staff participants; (ii) identify potential risks to implementation fidelity through staff exposure and responsiveness to the DCM-NR procedure and feedback or contamination across experimental groups; (iii) assess the acceptability, mechanisms, and any unintended consequences of the trial, and (iv) estimate the variance of outcome measures, to assist with subsequent sample size calculations.

\section{Methods}

\section{Design}

A mixed-methods cluster-randomised study design was used.

\section{Participants}

A convenience sample of 41 staff members was recruited from four neurorehabilitation wards in a hospital in the North West of England. One ward provided specialized care for individuals affected by neurological conditions. The second cared for patients who were experiencing cardiovascular difficulties such as stroke or vascular dementia. The third ward cared for people in a 'hyper acute' phase who required a high level of care following neurological trauma. The final ward cared for people in a 'post-acute' phase, who were able to engage in cognitive and physical rehabilitation following neurological trauma. Inclusion criteria for staff were that they had to be: of working age; employed on a contract with the participating hospital; based solely on one of the four participating wards; expected to still be working on that ward at follow up; and employed in a role that involved interacting with patients.

Randomisation to groups was done at the ward level, before any baseline assessments had been completed. This was done by first sealing the name of each of the four participating wards into a separate opaque envelope, and then placing all envelopes into a bag. Allocation to the experimental condition (DCM-NR) was done by drawing two of the sealed envelopes from the bag. The wards named in the remaining two envelopes were then allocated to the control condition (continue with care as usual, with DCM-NR provided after the study had finished). The names of the wards allocated to each condition were given to the staff members who were performing the mapping, but not to the researchers who were collecting baseline and follow-up data.

\section{Outcome measures}

Quality of Interactions Schedule (QUIS; Dean, Proudfoot \& Lindesay, 1993) 
The QUIS acted as a separate observational measure to assess the impact of DCM-NR on staff PCC behaviour. During each QUIS observation period, a researcher sat in an unobtrusive part of the ward, and recorded all interactions between patients and staff that occurred within their field of vision. A brief description of the verbal and non-verbal behaviours occurring during each interaction was recorded, along with details of the time that the interaction occurred, and which categories of people the interaction was between (e.g. patient and ward staff member). Each interaction was then rated as belonging to one of the following five categories: positive social, positive care, neutral, negative protective, or negative restrictive. Positive social interactions are defined as interactions typified by 'good, constructive, beneficial conversation and companionship' that is 'more than necessary' to complete the task (Dean, Proudfoot \& Lindesay, 1993, p.825). This is consistent with the conceptualisation of PCC as prioritising relationships and wellbeing over tasks (Edvardsson et al., 2008), and thus served as measures of PCC. Levels of agreement in how staff-patient interactions were categorised by the two researchers who performed the QUIS assessments reached a "near perfect" level of agreement (Cohen's K=.84, $p<.0001$; Landis and Koch, 1977) prior to the study start, indicating that the behavioural coding performed during the study was reliable.

\section{Approaches to Brain Injury Questionnaire (ABIQ)}

The Approaches to Dementia Questionnaire (ADQ; Lintern et al., 2000) was adapted for this study to assess attitudes to people with brain injury. The original ADQ includes 19 attitudinal items that reflect attitudes and beliefs towards people with dementia that are considered likely to promote good care (e.g. It is important for people with dementia to be given as much choice as possible in their daily lives.). Each item is scored from 1 (strongly disagree) to 5 (strongly agree), resulting in a total score ranging from 19-95. Nine items are reverse-scored, such that higher scores always indicate more positive attitudes. For the ABIQ, the word 'dementia' was replaced with '(a) brain injury', and the word 'residents' replaced with 'patients', in all relevant items. The item beginning with the phrase 'Once dementia develops in a person...' was also changed to 'Once a person has a brain injury...'. Cronbach's alpha for the ABIQ administered to 41 participants in the baseline phase of the study was .61.

\section{PCC Self-Efficacy}

This measure contained a list of 12 items describing PCC behaviours (e.g. Listen to the patient with empathy) taken from Leplage et al. (2007). Participants were instructed to rate their degree of confidence in performing each behaviour by responding to the question: 'Right now how certain are you that could...'. Participants were asked to record their response on a scale from $0-100$, where $0=$ Cannot do at all; $50=$ Moderately can do; and 100 $=$ Highly certain can do. The instructions and response scale were constructed using Bandura's guide for constructing a self-efficacy scale (Bandura, 2006). The average score of all responses was calculated and used for analysis. Cronbach's alpha for the PCC SelfEfficacy Scale administered to 41 participants in the baseline phase of the study was .82 .

\section{Procedure}


The research project was granted ethical approval by an NHS Research Ethics Committee (REC- North West - Greater Manchester Central Research Ethics Committee, 16/NW/0263). All participants provided written informed consent before taking part in the study.

Staff members were informed about the project through group staff briefings, one-toone meetings with the researchers, or from copies of the participant information sheet available on the ward. Staff members who wanted to take part were invited to attend a one-toone session with a researcher, in which individual baseline outcome measure data (selfreported ABIQ and PCC self-efficacy, as well as a written description of recent care provided, which was not included in this report) were collected.

Baseline QUIS observations then took place on each ward. For this, each ward was observed on two two-hour occasions, which is in line with recommendations for obtaining representative samples of data (Chenoweth and Jeon, 2007; Chenoweth et al., 2009). During observations, the researcher sat in an unobtrusive part of a communal bay and recorded details of all interactions that occurred between staff and patients within this time frame. Every time an interaction occurred between a patient and a member of staff, anonymous details of the interaction were noted, and it was coded as positive social, positive care, neutral, negative protective, or negative restrictive. Interactions occurring in private rooms or behind closed curtains were not recorded. As these data were collected at the level of the ward rather than the individual, staff members who were observed were not necessarily those who were participating in the study. Staff members and patients were given the opportunity to opt out of being observed, but none chose to do so.

Following baseline data collection, the DCM-NR intervention was delivered on the two experimental wards. No DCM-NR mapping or feedback sessions were provided for staff in the two control wards, who delivered care as usual. In line with DCM-NR protocol, prior to the start of mapping, inter-rater reliability maps were conducted for each pair of mappers to ensure that the minimum concordance level of $70 \%$ concordance had been achieved (Surr $\&$ Bonde-Nielsen, 2003). Each of the experimental wards was then mapped on two separate occasions at two different times of the day. Mappers were senior members of staff with previous experience of working on neuroscience wards. Mappers sat in an unobtrusive area, mainly out of sight of patients and staff, in a bay on the ward that maximised the number of observable patients. Each mapper observed up to eight patients at a time, and recorded the behaviour or activity that the patient was engaging in according to the pre-specified list of DCM-NR behaviour category codes (O'Hanlon et al, 2014) at five minute intervals. The patient's level of mood and engagement with that activity, and quality of the staff-patient interactions, were also coded according to Edition 1 of the Care Mapping Neuro Services ${ }^{\text {TM }}$ (DCM-NS ${ }^{\mathrm{TM}}$ ) User's Manual.

Data from the DCM-NR observations were then analysed as per the manual guidelines, and used to provide feedback for staff. For one of the experimental wards (from which 11 members of staff had been recruited), feedback sessions were provided approximately two weeks after the DCM-NR observations. Copies of a written feedback 
report were also made available for these staff, and discussed at a feedback meeting. The feedback report contained quantitative data relating to patient well-being, as well as qualitative information related to staff strengths, issues for the ward to consider and discuss as a team, and future actions. Although feedback sessions had been scheduled for the second experimental ward (from which 10 members of staff had been recruited), these did not occur before the end of the study due to unexpected staff shortages. In-line with intention-to-treat guidelines (Gupta, 2011), follow-up data were still collected for these participants and included in the analyses.

Following the DCM-NR intervention period, each of the participating wards received two two-hour follow-up QUIS observations, and individual follow-up data were collected from participating staff members. In addition to the measures collected at baseline, participants in the control group were asked to complete a short questionnaire to assess levels of contamination. This asked participants to report which (if any) other wards they had worked on since the start of the study; whether they had spoken to anyone from the experimental wards about care mapping; and what (if anything) they had heard about it.

Participants in the experimental group were asked to complete a short questionnaire to assess exposure, responsiveness to, and acceptability of, the intervention. This comprised questions relating to whether they had been on shift and/or observed during the mapping or QUIS observations; whether they had received feedback from the mapping; and their perceptions of the utility, relevance, appropriateness and feasibility of care mapping in their ward. Responses to the acceptability items were made on a 5-point scale, ranging from 'Not $\mathrm{X}$ at all' to 'Very X', where $\mathrm{X}$ was the descriptor word in each item (e.g. 'Worthwhile', 'Relevant', 'Appropriate').

Participants in the experimental group were also asked to take part in a short, semistructured interview to explore issues relating to the acceptability, mechanisms, and any unintended consequences of the DCM-NR intervention. For this, the interviewer used a topic guide to ask questions about their thoughts and feelings about being observed (during mapping and/or QUIS collection) and receiving feedback and action plans from the mapping, as well as any changes they had noticed in their own or others' behaviour. Some staff were also asked about their experiences of completing the outcome measures. Interviews lasted around 20 minutes and were audio recorded, and subsequently transcribed verbatim.

\section{Data analysis}

For QUIS data, the proportion of PCC behaviours (i.e. those coded as positive social) was compared to the proportion of all other types of behaviours (i.e. positive care; neutral; negative protective; and negative restrictive). Although the data points cannot be considered truly independent of one another (as individual members of staff present during each observation can each contribute several data points), $2 \times 2$ chi squared analyses were used to determine whether these proportions differed by group at each of the two time points. 
ADBI and PCC Self-Efficacy data were not normally distributed, and so nonparametric Mann-Whitney U tests were used to compare median scores in each phase between the experimental and control groups, and Wilcoxon signed-rank tests were used to compare pre and post phase scores for each group.

Verbatim transcripts of interview data were analysed using thematic analysis (Braun $\&$ Clarke, 2013). For this, segments of data relevant to the acceptability, potential mechanisms of change, or unintended consequences of the intervention were first given a descriptive code. These codes were then used to identify clusters of related points, which were grouped to form themes.

\section{Results}

\section{Recruitment and retention rates}

A total of 58 staff were briefed about the research study. Fifty-two (90\%) of these showed an interest in the study at the briefing by booking a time with a researcher to complete consent and baseline assessments. However, due to circumstances such as holidays, problems in shift patterns, and sickness, 11 (21\%) of the 52 potential participants did not enrol into the study. Forty one participants ( $71 \%$ of those briefed) were therefore recruited into the study. Twentyone of these participants were in the experimental group and 20 were in the control group.

Twenty-nine participants completed the follow-up measures (13 from the experimental group; 16 from the control group), resulting in a retention rate of $70.1 \%(61.9 \%$ for the experimental group; $80 \%$ for the control group). None of the participants actively withdrew from the study. Instead, participants were lost to follow up due to participants' shift patterns $(n=4)$, sickness $(n=3)$, annual leave $(n=2)$, job changes $(n=2)$, and time constraints $(\mathrm{n}=1)$ making it impossible to collect follow-up data within the timeframe of the study. The two groups did not differ from one another in terms of gender, age, ethnicity, occupation, or number of years of experience on the current ward or in healthcare (Table 1).

[Insert Table 1 about here]

\section{Exposure and responsiveness to mapping and feedback}

All scheduled DCM-NR mapping sessions were completed. Seven (54\%) of the 13 participants in the experimental group reported being on shift during the DCM-NR mapping, with one additional participant stating that they were unsure. Four of these seven ( $31 \%$ of total mapped) reported that they had been observed by a care mapper, one that they had not been observed, and two were unsure. Ten (77\%) of the 13 participants in the experimental group reported that they had been observed during either DCM-NR or QUIS mapping, meaning that most participants had some experience of having their behaviour observed during the study.

Three of the 13 participants in the experimental group worked on the ward that had not received a feedback session. Of the 10 participants from the ward that did receive 
feedback, three (23\% of the total group) reported that they had attended a feedback session: two of these had read the action plan, one of whom had also been involved in developing the action plan. All of the staff who had read the written report and action plan and/or attended the feedback session rated each of them as 'very useful' (on a 5-point scale ranging from 'not useful at all' to 'very useful'). The two staff who had the read the written report and action plan also reported that they 'fully understood' them (on a 5-point scales ranging from 'did not understand at all' to 'fully understood), indicating high levels of responsiveness. However, both of these staff members reported that they had not yet implemented any actions from the action plan.

\section{Contamination}

Of the 16 participants in the control condition, four (25\%) reported that they had worked on another ward during the study, but none of these had worked on either of the two experimental condition wards. Two (13\%) participants did report having heard about care mapping, but were only aware that observations were happening, and did not report any specific details. None of the 16 participants reported having spoken with anyone from the experimental condition wards during the study.

\section{Acceptability}

Participants in the experimental group reported that care mapping on the wards was very 'Worthwhile' $(\mathrm{M}=4.67, \mathrm{SD}=.78)$, that care mapping was very 'Relevant' to their clinical practice $(\mathrm{M}=4.58, \mathrm{SD}=.67)$, very 'Appropriate' for their ward $(\mathrm{M}=4.75, \mathrm{SD}=.45)$, and that the environment was 'Set up' for care mapping $(\mathrm{M}=4.25, \mathrm{SD}=.87)$.

Analysis of the qualitative interview data revealed that, whilst some staff initially felt a little apprehensive of care mapping, being observed, and/or receiving feedback, they was generally seen as positive and worthwhile endeavours, and most people soon got used to them.

I think people understand it more now, erm, and, like, if you came, next year and did it again, and it was all the same staff, I don't think they'd even, they wouldn't be phased by it or they'd, erm, I think most staff would welcome it, yeah. [...] Yeah, 'cos it, it's a positive thing, and I think everyone understands that now, yeah . (p22)

Whilst most staff were indifferent to who was doing the observing, one person felt that it would have been more intimidating to have been observed by someone who was not known to the staff, and another felt they might have been more nervous if observed by a consultant when doing something difficult.

I think it's probably more, I don't know if the words are right, it was intimidating if you don't know these people, you know? [...] But yeah, so, like I say I've known him [the mapper] for a long time and he's ok, you know? (p35) 
Those staff that talked about the outcome measures reported them to be acceptable, although a couple of staff did state the desire to add comments to explain why they gave certain answers, or under which conditions their answers might differ.

I'd like to elaborate more on some ... Like, for example, the one about, erm, people with a brain injury are very much like children: well no, they're not, but I've put agree, because they need routine, they need stability, they need constant reassurance, they need everything that a child needs, but they're not a child and we don't treat them like children, but we approach it in a similar way. But I just put agree. Well that might look like I think, I think they're like children. That's just one example. (p22)

\section{Mechanisms of change}

Some staff had noticed specific positive changes in their own or others' care behaviour, and others hadn't. Reasons given for why no change had occurred included: low staffing levels making change hard to implement; not having received the feedback; and a sense that no change was needed as the care that they were providing was already good.

I know that I do a good, that I do a good job, so I don't need to ... do anything different really. (p28)

Staff reported that the feedback was useful in terms of giving them insights into how their patients feel, and for making them question some of the everyday behaviours, habits and routines that that often stopped thinking about.

It does make you think about what you do every day. You can't see the wood for the trees, you come in every day, you do the same job, same routine, and if there's bad habits, you don't notice them because you're doing it every day. So if an outsider comes in and observes that and nips it in the bud, then ... it can only be good can't it. (p22)

They recognised the importance of having positive feedback for raising morale, and giving people the confidence to continue good care.

Yeah, definitely because that [positive feedback] allows you ... to realise what, what needs to be done, not in a, a negative way but, like if you know that you're doing this then right ok, this is what you're doing so continue to do that, yeah. (p40)

Several staff described the importance and benefits of being given the feedback verbally, and of being able to discuss it with other colleagues, suggesting that this would make it easier to digest, and more likely that people will take the information on board to make changes.

...better than, you know if you give someone a piece of paper and ask them to read it, they might forget or they get busy, and they never read it properly, you know? So if you ... it's interacting. (p32) 
There was a suggestion that action planning meetings would be useful for determining how to address the feedback. In particular, participants felt that this would prevent people from forgetting the feedback, or only focussing on the positive aspects. They also felt that having a concrete list of actions to take would make change more likely to occur.

I think if we have another meeting and actually say, bullet points, this is what we need to work on, then people might be more proactive in working on it. But because the feedback session, it's not vague but it, it mentions everything else, whereas if it's just about that, I think that would help. (p22)

...people interpret things differently. So unless you actually say to some people, right well we need to improve this, this and this by doing this, this and this, some people won't do it. (p22)

\section{Unintended consequences}

Unintended consequences of the study were largely related to staff behaviour when being observed. Some staff explained how they or others tried to 'stay away' from bays that were being observed; that some staff behaved more positively than usual when being observed; or that their behaviour felt less natural than usual.

Erm, when you're being watched, it feels like you ... it's being a bit forced, when you're with the patients. It's something I'd do anyway but when I knew I was being watched, I was like, oh I feel like I'm gonna have to go over it, do you know? but I would have done it anyway, but because you was there, it was like, you had to make a point of it, if that makes sense. (p10)

However, there was a general sense that most staff that any such effects on behaviour were relatively short-lived, as people soon got used to being observed, forgot about it, or were too busy to change their behaviour.

I think I was a bit nervous, because, erm, because somebody was watching me, erm, but, erm, I think after a while I just forgot, and just got on with my job, 'cos I'm so busy anyway. (p19)

Finally, whilst the importance of receiving positive feedback was widely acknowledged by staff, one participant did report that this focus on the positives did stop her from thinking about the things that need to change.

You think of the positives and you don't think of what needs improving, I'll be honest. (p22)

\section{Outcome Measures}

PCC

Examination of the counts across each QUIS observation session reveal considerable variation in the proportion of each interaction type, even within the same ward in the same 
phase of the study. For instance, at baseline, $69 \%$ of interactions on experimental ward 2 were classed as positive social in the first observation, compared with just $44 \%$ in the second observation session: a difference of $25 \%$ (Table 2).

[Insert Table 2 about here]

At baseline, there was no signification association between interaction type and group, $\chi^{2}(1)=.79, p=.37$. However, there was a significant association between group and interaction type at follow-up, $\chi^{2}(1)=16.18, p<.001$. Z-test comparisons of the column proportions showed that, whilst both groups made a higher proportion of non-PS behaviours to PS behaviours, a higher proportion of the PS responses were made by the experimental group relative to the control group (Table 3).

[Insert Table 3 about here]

Attitudes to People with Brain Injury

One participant in the experimental group had missed three items on the ABIQ, and so these missing responses were replaced with the participant's mean score for the other 16 items. Mann-Whitney U tests showed that median ABIQ scores did not differ between the experimental and control group at either baseline $(\mathrm{U}=90.5, p=.55)$ or follow up $(\mathrm{U}=74.0$, $p=.19)$. Wilcoxon signed-rank tests showed that the scores did not change from baseline to follow-up for either the experimental $(\mathrm{Z}=.95, p=.34)$ or the control group $(\mathrm{Z}=.13, p=.89)$. The median and range of scores in each condition are shown in Table 4.

PCC self-efficacy Median self-efficacy scores did not differ between the experimental and control group at either baseline $(\mathrm{U}=95.5, p=.71)$ or follow-up $(\mathrm{U}=79.5, p=.28)$. Whilst the experimental group showed no change in SE score across phases $(\mathrm{Z}=.28, p=$ .78), median SE scores in the control group actually increased from the pre to post phase $(\mathrm{Z}=$ $2.25, p=.025)$. The median and range of scores in each condition are shown in Table 4.

[Insert Table 4 about here]

\section{Discussion}

The aims of this study were to conduct pilot and feasibility testing of a cluster-randomised controlled trial to assess the effectiveness of DCM-NR at increasing levels of PCC in neurorehabilitation settings. Seventy-one percent of staff briefed about the study consented to participate, although $30 \%$ of these were lost to follow up. Whilst DCM-NR and the trial were generally acceptable to participants, levels of exposure were relatively low. There was no evidence of contamination between conditions, although a quarter of staff did report working on at least one other ward during the timeframe of the study. A higher proportion of PCC behaviour was observed in the experimental group compared to the control group at followup, although this was due to a decrease in PCC behaviour in the control group that was not observed in the experimental group. High levels of variability in interaction types were also 
observed within conditions. Neither group showed a change in attitudes towards people with brain injury, and participants in the control, but not the experimental, group showed an increase in PCC-self efficacy over time. Taken together, the findings suggest that this clusterrandomised trial design is acceptable, and also highlights modifications to the protocol and outcome measures that could increase the success and theoretical value of a future large-scale study.

The high recruitment rate observed in the study is particularly encouraging given that no financial or other incentive was offered for participation. However, the proportion of participants who were lost to follow-up is indicative of the challenges of working within a busy hospital environment. Future large-scale studies of this type should either plan for similar retention rates in their initial recruitment, or consider additional strategies for increasing retention, such as the provision of financial incentives or by using up-front scheduling to book in follow-up data collection sessions (Page \& Persch, 2013). In addition, future studies should bear in mind that the actual number of participants who were exposed to the intervention was much lower than of those recruited. This was partly due to unexpected staff shortages, resulting in one ward not being able to deliver any feedback sessions in time, and was also due to the practical challenges that have previously been noted (O'Hanlon et al., 2014) of scheduling feedback sessions when a sufficient number of staff could attend. As DCM is implemented at a system level, every staff member does not need to be directly involved with every component. Nevertheless, greater levels of exposure to the feedback are clearly desirable. In order to maximise exposure levels, and given the pressures on staff time, future intervention studies would therefore benefit from costing in staff time for performing mapping and feedback rather than relying on existing staff resources.

Most staff were positively disposed to DCM, and found all elements of the trial to be generally acceptable: a factor considered important for intervention fidelity (Carroll et al., 2007). These findings extend those of Westbrook et al. (2013) to show that DCM-NR is acceptable in an intervention context, as well as showing that the trial itself is well-tolerated by staff. As some staff reported feeling initially unnatural, self-conscious, or avoidant during observation periods, future studies might benefit from adding an initial settling-in period to each observation session, the data from which could subsequently discarded from the analysis.

Although this study was not powered to show the effects of the intervention, it is encouraging to see that a significantly greater proportion of PCC was observed in the experimental group compared to the control group at follow up but not baseline. These group differences occurred due to the control group showing a decline in PCC over time whilst the experimental group remained stable: perhaps reflecting the additional effects of participants acclimatising to being observed, and/or the uncharacteristically high staffing shortages occurring in the hospital during the time of the intervention and follow-up period, which almost reached 'major incident status'. Of note for future studies is the relatively high level of variability in interaction types observed across different observation sessions for the same 
ward in the same phase of the study: perhaps reflecting different staff on shift, or specific demands of the day. This high level of variability suggests that additional, or longer, periods of QUIS observation may be needed in order to gain more reliable and representative assessments of the care behaviour occurring at each time point.

No significant effects were seen on staff attitude to brain injury scores, although there was a trend towards an improvement in these scores in the experimental group that was not observed in the control group. PCC self-efficacy scores, however, changed in the opposite direction to what was expected: with the control, but not the experimental, group showing a significant increase in self-efficacy over time. The increase in self-efficacy seen in the control group may just be an effect of the timing or repeated nature of testing. One potential explanation for the lack of a similar increase in self-efficacy in the experimental group might be that one effect of highlighting required improvements in care to staff might be that staff initially feel less competent at delivering PCC. Indeed, receiving feedback that performance does not meet an expected standard has been linked to initial reductions in self-efficacy (Ilgen \& Davis, 2000), at least until the individual has had opportunity to gain sufficient opportunity to restore confidence in their ability (Dahling \& Ruppel, 2016). Future studies assessing the impact of DCM-NR should therefore track self-efficacy over a longer period so that both short and longer term effects can be assessed.

The qualitative interviews in this study also revealed other factors that may influence the effectiveness of DCM-NR, and thus might be worth assessing in a future full-scale trial. For instance, the beneficial effects of positive feedback on staff morale were mentioned by some participants, and could be worth assessing as a possible mediator of PCC in a full-scale trial. A number of participants also mentioned how an action planning meeting after the feedback sessions would be helpful for enabling change. As well as enabling ownership of the action plans (Brooker, 1998), study participants felt that such meetings would mean that explicit instructions could be provided to those staff that need them, and also prevent staff members from only focussing on the positive feedback received. Given that there was some evidence for increased PCC without having had action planning meetings, a future large-scale trial could compare the effectiveness of DCM-NR with and without an action-planning in order to formally assess the additional utility of this DCM-NR component.

This feasibility and pilot study has also led to the identification of some design features that would benefit from being changed in a future full-scale. First, the reliability of the attitude measure was relatively low, suggesting that some refinement to this scale is needed. Indeed, Cheston et al. (2016) have recently argued that some of the language used in the items of the Approaches to Dementia questionnaire, from which the ABIQ was developed, may need adapting to be more consistent with contemporary understanding of dementia care. Similar changes may also therefore be required to ensure that the questionnaire accurately reflects attitudes to brain injury that are considered most conducive to PCC. In addition, it is notable that levels of PCC, self-efficacy, and attitudes were relatively high in both groups at baseline, which might limit the amount of change that was 
possible. More sensitive tests of the effectiveness of DCM-NR might therefore be possible by recruiting participants with lower baseline levels of PCC.

There were some other limitations to this study. First, as mentioned above, only one of the two wards allocated to the intervention group received the planned feedback session before follow up data were collected. This means that data relating to the effectiveness of DCM-NR are likely to represent an under-estimate of their true value. It also meant that fewer participants were able to report their experiences of these feedback sessions. This inability to schedule this feedback session does however highlight the practical difficulties that staff face when trying to adhere to a trial protocol alongside the real-life demands of the clinical environment. Second, the ethnic diversity of participants was relatively low, with 96\% of staff who reported their ethnicity identifying as either 'White British' or 'White Other'. Finally, interactions that occurred in private rooms or behind closed curtains were not observed during the intervention, and so did not contribute to PCC scores. Whilst this observational strategy is in line with methodological guidelines (Dean, Proudfoot \& Lindesay, 1993), and served to maximise the number of interactions that were observed and minimise intrusiveness to patients, it did mean that some of the more intimate types of care behaviours were not included in the intervention. Future interventions could address this by dedicating time to observing one-to-one care interactions in the private rooms or curtained areas of patients who are comfortable with this in order to ensure that levels of PCC can be assessed and improved for a wider range of care situations.

In conclusion, this study suggests that a cluster-randomised DCM-NR intervention is acceptable and potentially efficacious in increasing levels of PCC in neurorehabilitation settings. The study design is feasible, but could be made more effective by increasing retention levels; costing in staff time to deliver and monitor feedback sessions; collecting additional observation outcome data; refining the measure of attitudes; and by recruiting from a population with lower baseline levels of PCC behaviour, attitudes and self-efficacy. The scientific value of the study could also be increased through the addition of outcome measures and experimental conditions in order to identify important mediators and moderators of effectiveness. 
Acknowledgements: We wish to thank Dr Charlotte Richardson for the contributions that she made to study design, data collection, and drafting of the manuscript. We would also like to thanks all staff and patients at the participating hospital for their support and/or involvement with this study.

Declaration of Interest Statement: None of the authors have any potential conflicts of interest to report. 


\section{References}

Bandura, A. 2006. Guide for constructing self-efficacy scales, in: Pajares, F. \& Urdan, T. (Eds.), Self-efficacy Beliefs of Adolescents. Information Age Publishing: Connecticut.

Bradford Dementia Group (2005). DCM 8. User's manual: The DCM method (8th Edn.) Bradford: University of Bradford

Brooker, D. (2005). Dementia care mapping: a review of the research literature. The Gerontologist, 45, 11-18.

Carroll, C., Patterson, M., Wood, S., Booth, A., Rick, J. \& Balain, S. (2007). A conceptual framework for implementation fidelity. Implementation Science, 2, 1-9.

Chenoweth, L \& Jeon, Y-H. (2007). Determining the efficacy of Dementia Care Mapping as an outcome measure and a process for change: A pilot study. Aging \& Mental Health, 11, 237-245. doi: 10.1080/13607860600844226.

Chenoweth, L., King, M. T., Jeon, Y. H., Brodaty, H., Stein-Parbury, J., Norman, R, Haas, M., \& Luscombe, G. (2009). Caring for Aged Dementia Care Resident Study (CADRES) of person-centred care, dementia-care mapping, and usual care in dementia: a cluster-randomised trial. Lancet Neurology, 8, 317-325. doi: 10.1016/s1474-4422(09)70045-6.

Cheston, R., Hancock, J., \& White, P. (2016). A cross-sectional investigation of public attitudes toward dementia in Bristol and South Gloucestershire using the approaches to dementia questionnaire. International Psychogeriatrics, 28, 1717-24.

Dahling, J.J. \& Ruppel, C.L. (2016). Learning goal orientation buffers the effects of negative normative feedback on test self-efficacy and reattempt interest. Learning and Individual Differences, 50, 296-301.

Dane, A. V., \& Schneider, B.H. (1998). Program integrity in primary and early secondary prevention: are implementation effects out of control? Clinical Psychology Review, $18,23-45$.

Dean, R., Proudfoot, R., \& Lindesay, J. (1993). The Quality of Interactions Schedule (QUIS): Development, reliability and use in the evaluation of two domus units. International Journal of Geriatric Psychiatry, 8, 819-826.

Department of Health. (2005). The National Service Framework for Long-term Conditions. Retrieved from https://www.gov.uk/government/publications/quality-standards-forsupporting-people-with-long-term-conditions 
Godin, G., Bélanger-Gravel, A., Eccles, M. \& Grimshaw, J. (2008). Healthcare professionals' intentions and behaviours: A systematic review of studies based on social cognitive theories. Implementation Science, 3, 1-12.

Gupta, S.K. (2011). Intention-to-treat- concept: a review. Perspectives in Clinical Research, 2, 109-112.

Ilgen, D.R. \& Davis, C.A. (2000). Bearing bad news: reactions to negative performance feedback. Applied Psychology: An International Review, 49, 550-565.

Kuhn, D., Ortigata, A., \& Kasayka, R.E. (2000). Dementia care mapping: an innovative tool to measure person-centred care. Alzheimer's Care Quarterly, 1, 7-15.

Landis, R. J., \& Koch, G. G. (1977). The Measurement of Observer Agreement for Categorical Data. Biometrics, 33, 159-174.

Leplege, A., Gzil, F., Cammelli, M., Lefeve, C., Pachoud, B., \& Ville, I. (2007). Person centredness: conceptual and historical perspectives. Disability and Rehabilitation, 29, 15551565 .

Lintern, T., Woods, B., \& Phair, L. (2000). Training is not enough to change care practice. Journal of Dementia Care, 8, 15-17.

Martin, G., \& Younger, D. (2001). Person-centred care for people with dementia: A quality audit approach. Journal of Psychiatric and Mental Health Nursing, 8, 443-448. doi:http://dx.doi.org/10.1046/j.1351-0126.2001.00427.x

McIntosh, C. J., Westbrook, J., Sheldrick, R., Surr, C., \& Hare, D. J. (2012). The feasibility of Dementia Care Mapping (DCM) on a neurorehabilitation ward. Neuropsychological Rehabilitation, 22, 920-941.

Medical Research Council (2006). Developing and evaluating complex interventions: new guidance Retrieved from https://www.mrc.ac.uk/documents/pdf/complexinterventions-guidance

O'Hanlon, K., Leigh, A., Sheldrick, R., Surr, C., \& Hare, D. J. (2015). The feasibility and validity of care mapping in the clinical neurosciences. Neuropsychological Rehabilitation, 25, 818-840. doi: 10.1080/09602011.2014.978873

Page, S. J., \& Persch, A. C. (2013). Recruitment, Retention, and Blinding in Clinical Trials. American Journal of Occupational Therapy, 67, 154-161. 
Surr, C., \& Bonde-Nielsen, E. (2003). Inter-rater reliability in DCM. Journal of Dementia Care, 11, 33-36.

Westbrook, J, L., McIntosh, C, J., Sheldrick, R., Surr, C., \& Hare, D, J. (2013). Validity of Dementia Care Mapping on a neuro-rehabilitation ward: Q-methodology with staff and patients. Disability and Rehabilitation, 35, 1652-1659. doi:

$10.3109 / 09638288.2012 .748839$ 
Table 1. Characteristics of participants who provided baseline and follow-up data. All between-group comparisons were non-significant $(p>.05)$, using Fisher's exact test for gender, ethnicity (White British vs. all other ethnicities), and occupation (Clinical support worker vs. Staff nurse vs. Sister vs. all other), and t-test for age and years on current ward and in healthcare.

\begin{tabular}{|c|c|c|}
\hline & $\begin{array}{l}\text { Experimental group } \\
\left(n=13^{*}\right)\end{array}$ & $\begin{array}{l}\text { Control group } \\
(n=16)\end{array}$ \\
\hline Gender: number (\%) & Female: $10(77 \%)$ & Female: $13(81 \%)$ \\
\hline Mean age (SD): years & $38.7(13.1)$ & $35.9(11.1)$ \\
\hline \multicolumn{3}{|l|}{ Ethnicity: number (\%) } \\
\hline White British & $10(77 \%)$ & $15(94 \%)$ \\
\hline Black British & 0 & $1(6 \%)$ \\
\hline White Other & $2(15 \%)$ & 0 \\
\hline Not specified & $1(8 \%)$ & 0 \\
\hline \multicolumn{3}{|l|}{ Occupation: number (\%) } \\
\hline Clinical support worker & $7(54 \%)$ & $9(56 \%)$ \\
\hline Staff nurse & $2(15 \%)$ & $4(25 \%)$ \\
\hline Sister & $1(8 \%)$ & $2(13 \%)$ \\
\hline Assistant practitioner & 0 & $1(6 \%)$ \\
\hline Advanced nurse practitioner & $1(8 \%)$ & 0 \\
\hline Occupational Therapist & $1(8 \%)$ & 0 \\
\hline Housekeeper & $1(8 \%)$ & 0 \\
\hline $\begin{array}{l}\text { Mean (SD): years worked on } \\
\text { current ward }\end{array}$ & $2.35(1.2)$ & $4.02(3.0)$ \\
\hline $\begin{array}{l}\text { Mean (SD): years' experience } \\
\text { in healthcare }\end{array}$ & $12.69(10.6)$ & $9.85(8.7)$ \\
\hline
\end{tabular}

* One of these 13 participants did not complete questionnaire items relating to responsiveness or acceptability, and did not take part in an interview. 
Table 2: Frequency of QUIS interaction types for each ward, in each observation session at baseline. For analysis, the numbers of each observation type were summed across four observation sessions for each group: two for each ward. Data highlighted in bold indicate those used in the chi square tests.

\begin{tabular}{llrrrrrrr}
\hline & \multicolumn{6}{c}{ Number $($ and \%) } & of interactions coded in each QUIS category \\
Ward & Session & PS & PC & N & NP & NR & NonPS & TOTAL \\
\hline Experimental & 1 & 20 & 31 & 4 & 0 & 0 & 35 & 55 \\
Ward 1 & & $(36 \%)$ & $(56 \%)$ & $(7 \%)$ & $(0 \%)$ & $(0 \%)$ & $(64 \%)$ & \\
& 2 & 7 & 17 & 7 & 0 & 0 & 24 & 31 \\
& & $(23 \%)$ & $(55 \%)$ & $(23 \%)$ & $(0 \%)$ & $(0 \%)$ & $(77 \%)$ & \\
Experimental & 1 & 41 & 16 & 2 & 0 & 0 & 18 & 59 \\
Ward 2* & & $(69 \%)$ & $(27 \%)$ & $(3 \%)$ & $(0 \%)$ & $(0 \%)$ & $(31 \%)$ & \\
& 2 & 11 & 12 & 1 & 1 & 0 & 14 & 25 \\
& & $(44 \%)$ & $(48 \%)$ & $(4 \%)$ & $(4 \%)$ & $(0 \%)$ & $(56 \%)$ & \\
\hline Experimental Group: & $\mathbf{7 9}$ & 76 & 14 & 1 & 0 & $\mathbf{9 1}$ & 170 \\
Total & & $(\mathbf{4 6 \% )}$ & $(45 \%)$ & $(8 \%)$ & $(1 \%)$ & $(0 \%)$ & $(\mathbf{5 4 \%})$ & \\
\hline Control & 1 & 15 & 23 & 0 & 0 & 0 & 23 & 38 \\
Ward 1 & $(39 \%)$ & $(61 \%)$ & $(0 \%)$ & $(0 \%)$ & $(0 \%)$ & $(61 \%)$ & \\
& 2 & 6 & 24 & 0 & 0 & 0 & 24 & 30 \\
Control & 1 & $(20 \%)$ & $(80 \%)$ & $(0 \%)$ & $(0 \%)$ & $(0 \%)$ & $(80 \%)$ & \\
Ward 2 & 22 & 14 & 0 & 0 & 0 & 14 & 36 \\
& 2 & $(61 \%)$ & $(39 \%)$ & $(0 \%)$ & $(0 \%)$ & $(0 \%)$ & $(39 \%)$ & \\
& & 18 & 23 & 1 & 1 & 0 & 25 & 43 \\
\hline Control Group: Total & $(42 \%)$ & $(53 \%)$ & $(2 \%)$ & $(2 \%)$ & $(0 \%)$ & $(58 \%)$ & \\
\hline
\end{tabular}

$\mathrm{PS}=$ Positive Social; $\mathrm{PC}=$ Positive Care $\mathrm{N}=$ Neutral; NP = Negative Protective; $\mathrm{NR}=$ Negative Restrictive. *Experimental Ward 2 was the ward that did not receive a feedback session. 
Table 3: Frequency of QUIS interaction types for each ward, in each observation session at follow-up. For analysis, the numbers of each observation type were summed across four observation sessions for each group: two for each ward. Data highlighted in bold indicate those used in the chi square tests.

\begin{tabular}{llrrrrrrr}
\hline & & \multicolumn{6}{c}{ Number (and \%) } & of interactions coded in each QUIS category \\
Ward & Session & PS & PC & N & NP & NR & NonPS & TOTAL \\
\hline Experimental & 1 & 18 & 25 & 0 & 0 & 0 & 25 & 43 \\
Ward 1 & & $(42 \%)$ & $(58 \%)$ & $(0 \%)$ & $(0 \%)$ & $(0 \%)$ & $(58 \%)$ & \\
& 2 & 13 & 25 & 0 & 0 & 0 & 25 & 38 \\
& & $(34 \%)$ & $(66 \%)$ & $(0 \%)$ & $(0 \%)$ & $(0 \%)$ & $(66 \%)$ & \\
Experimental & 1 & 11 & 30 & 1 & 0 & 0 & 31 & 42 \\
Ward 2* & & $(26 \%)$ & $(71 \%)$ & $(2 \%)$ & $(0 \%)$ & $(0 \%)$ & $(74 \%)$ & \\
& 2 & 12 & 5 & 2 & 0 & 0 & 7 & 19 \\
& & $(63 \%)$ & $(26 \%)$ & $(11 \%)$ & $(0 \%)$ & $(0 \%)$ & $(37 \%)$ & \\
\hline Experimental Group: & $\mathbf{5 4}$ & 85 & 3 & 0 & 0 & $\mathbf{8 8}$ & 142 \\
Total & & $(\mathbf{3 8 \%})$ & $(60 \%)$ & $(2 \%)$ & $(0 \%)$ & $(0 \%)$ & $(\mathbf{6 2 \%})$ & \\
\hline Control & 1 & 1 & 45 & 5 & 2 & 1 & 53 & 54 \\
Ward 1 & $(2 \%)$ & $(83 \%)$ & $(9 \%)$ & $(4 \%)$ & $(2 \%)$ & $(98 \%)$ & \\
& 2 & 16 & 30 & 0 & 1 & 0 & 31 & 47 \\
& & $(34 \%)$ & $(64 \%)$ & $(0 \%)$ & $(2 \%)$ & $(0 \%)$ & $(66 \%)$ & \\
\hline Control & 1 & 5 & 16 & 0 & 0 & 0 & 16 & 21 \\
Ward 2 & & $(24 \%)$ & $(76 \%)$ & $(0 \%)$ & $(0 \%)$ & $(0 \%)$ & $(76 \%)$ & \\
& 2 & 2 & 17 & 2 & 0 & 0 & 19 & 21 \\
& & $(10 \%)$ & $(81 \%)$ & $(10 \%)$ & $(0 \%)$ & $(0 \%)$ & $(90 \%)$ & \\
\hline Control Group: Total & $\mathbf{2 4}$ & 108 & 7 & 3 & 1 & $\mathbf{1 1 9}$ & 143 \\
& & $(\mathbf{1 7 \%})$ & $(76 \%)$ & $(5 \%)$ & $(2 \%)$ & $(1 \%)$ & $(\mathbf{8 3 \%})$ & \\
\hline
\end{tabular}

PS = Positive Social; $\mathrm{PC}=$ Positive Care $; \mathrm{N}=$ Neutral $; \mathrm{NP}=$ Negative Protective $; \mathrm{NR}=$ Negative Restrictive. *Experimental Ward 2 was the ward that did not receive a feedback session. 
Table 4: Attitude and self-efficacy data for participants in each group at baseline and followup.

\begin{tabular}{lllll}
\hline & \multicolumn{2}{c}{ Experimental Group (n=13) } & & \multicolumn{2}{c}{ Control Group (n=16) } \\
Measure & $\begin{array}{c}\text { Baseline } \\
\text { Median } \\
\text { (range) }\end{array}$ & $\begin{array}{c}\text { Follow-Up } \\
\text { Median } \\
\text { (range) }\end{array}$ & $\begin{array}{c}\text { Baseline } \\
\text { Median } \\
\text { (range) }\end{array}$ & $\begin{array}{c}\text { Follow-Up } \\
\text { Median (range) }\end{array}$ \\
\hline $\begin{array}{l}\text { ABIQ (total score: } \\
\text { possible range 19- }\end{array}$ & $80.0(68-88)$ & $82.0(68-87)$ & $78.5(68-87)$ & $78.5(69-87)$ \\
$\begin{array}{l}\text { 95) } \\
\text { PCC self-efficacy } \\
\text { (mean score/ 100) }\end{array}$ & $87.5(77-100)$ & $87.5(78-100)$ & $87.1(71-98)$ & $90.8(65-100)$ \\
\hline
\end{tabular}

